\title{
A Phase Shift Demodulation Technique: Verification and Application in Fluorescence Phase Based Oxygen Sensors
}

\author{
Chuanwu JIA ${ }^{1}$, Jun $\mathrm{CHANG}^{1 *}$, Fupeng WANG ${ }^{1}$, Hao JIANG ${ }^{1}$, \\ Cunguang $\mathrm{ZHU}^{2}$, and Pengpeng $\mathrm{WANG}^{2}$
}

\author{
${ }^{1}$ School of Information Science and Engineering and Shandong Provincial Key Laboratory of Laser Technology and \\ Application, Shandong University, Jinan, 250100, China \\ ${ }^{2}$ School of Physics and Technology, University of Jinan, Jinan, 250022, China \\ *Corresponding author: Jun CHANGＥ-mail: changjun@sdu.edu.cn
}

\begin{abstract}
A phase shift demodulation technique based on subtraction capable of measuring 0.03 phase degree limit between sinusoidal signals is presented in this paper. A self-gain module and a practical subtracter act the kernel parts of the phase shift demodulation system. Electric signals in different phases are used to verify the performance of the system. In addition, a new designed optical source, laser fiber differential source (LFDS), capable of generating mini phase is used to further verify the system reliability. R-square of 0.99997 in electric signals and R-square of 0.99877 in LFDS are achieved, and 0.03 degree measurement limit is realized in experiments. Furthermore, the phase shift demodulation system is applied to the fluorescence phase based oxygen sensors to realize the fundamental function. The experimental results reveal that a good repetition and better than $0.02 \%$ oxygen concentration measurement accuracy are realized. In addition, the phase shift demodulation system can be easily integrated to other applications.
\end{abstract}

Keywords: Phase shift detection system; self-gain, subtracter; LFDS; phase based oxygen sensor

Citation: Chuanwu JIA, Jun CHANG, Fupeng WANG, Hao JIANG, Cunguang ZHU, and Pengpeng WANG, "A Phase Shift Demodulation Technique: Verification and Application in Fluorescence Phase Based Oxygen Sensors," Photonic Sensors, 2016, 6(2): $169-176$.

\section{Introduction}

There is a considerable interest in the rapid and accurate measurement of phase shift for a variety of applications including biological, industrial, and environmental monitoring. Phase shift is a common parameter in the field of industrial measurement and controlling. Meanwhile, it is one of the basic tasks of the engineering signal analysis. In the practical work, we often need to measure the phase shift between two signals with the same frequency to solve variously practical problems. As the phase detection technique applied to scientific research, production practice, and other fields, the requirement of the phase detection technology turns to high precision and high intelligent. The main methods of phase shift measurement contain the variable delay-line method [1], phase-locked method [2], correlation method [3, 4], and the method of over-zero time [5]. In this paper, a system based on the subtraction measuring the phase shift is proposed. However, when most methods require the sophisticated equipment and careful calibration procedures, this phase shift demodulation system

Received: 21 January 2016 / Revised: 8 March 2016

(C) The Author(s) 2016. This article is published with open access at Springerlink.com

DOI: $10.1007 / \mathrm{s} 13320-016-0303-2$

Article type: Regular 
based on subtraction just relies on simple and cheap instrumentation and offers high sensitivity and fast response time.

Measurement of phase shift is different from the traditional detection of the voltage signal and current signal. First of all, phase difference is attached to the voltage and current signals. How to eliminate the changes in the voltage, current, and frequency is an important aspect of the phase detection. Secondly, measurement of the phase difference between two signals not only need to keep the same frequency of two signals, but also need to exclude the impact of other factors such as the amplitude of the two signals. Obviously, making the amplitude of the two input signals same seems very important. Up to now, the common method making the amplitude of two sinusoidal signals same is the self-gain circuit [6]. In order to modulate the amplitude of the signals, a self-gain module is set up.

The self-gain module is shown in Fig. 1. As
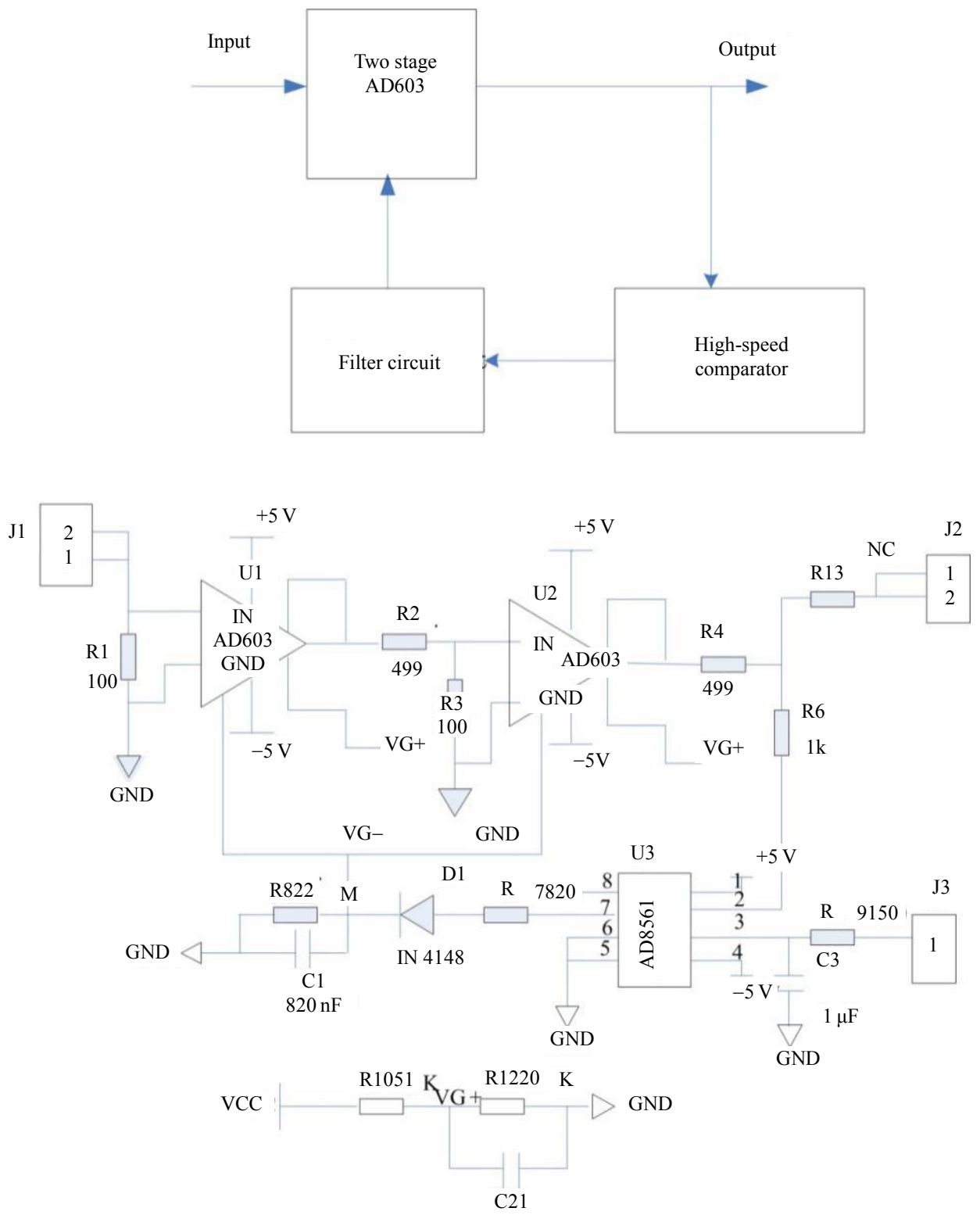

Fig. 1 Diagram of the self-gain module. 
shown in Fig. 1(a), the self-gain module mainly contains three parts: the two-stage amplifier part, the high-speed comparator, and the filter circuit. Two commercial chips AD603 (Analog Devices, USA) are involved to build the two-stage amplifier part. In addition, a high-speed comparator constructed by AD8561 (Analog Devices, USA) is used to compare the output signals of the second AD603 with the setting voltage of the comparator. Diodes and RC circuits are used to build the filter circuit and remove the noise ratio of the output signal of the comparator. In Fig. 1(b), all the chips are powered by \pm 5 voltage. $\mathrm{J} 1$ and $\mathrm{J} 2$ are the interface of the input and output signals, respectively. Meanwhile, J3 is the interface of the setting voltage and controls the amplitude of the output signal. In this design, a two-stage AD603 amplification is used whose whole range of the gain will be $-26 \mathrm{~dB}-54 \mathrm{~dB}$. The $\mathrm{AD} 603$ relies on the controlling voltage $\left[\left(\mathrm{VG}^{+}\right)-\right.$ $\left(\mathrm{VG}^{-}\right)$] to control the magnification. The range of the filter value $\mathrm{VG}^{-}$is $0-3.3 \mathrm{~V}$. VG+ can be expressed as (1):

$$
V G+=V_{C C} \frac{R_{12}}{R_{10}+R_{12}}=1.4 \mathrm{~V}
$$

So the range of the controlling voltage $\left.\left[\left(\mathrm{VG}^{+}\right)-\left(\mathrm{VG}^{-}\right)\right)\right]$is $-1.9 \mathrm{~V}-1.4 \mathrm{~V}$, and it contains the linear range of AD603 $(-500 \mathrm{mV}-500 \mathrm{mV})$. As a result, to make sure the value of the regulation voltage is in the range of the controlling voltage of $\mathrm{AD} 603(-500 \mathrm{mV}-500 \mathrm{mV})$ and make the AD603 in normal amplification, we set the VG+ to be $1.4 \mathrm{~V}$. $\mathrm{R} 9$ and $\mathrm{C} 3$ is a first-order low-pass filter, the cutoff frequency $\left(f_{c}\right)$ is

$$
f_{c}=\frac{1}{2 \pi R_{9} C_{3}}=719 \mathrm{~Hz} .
$$

The negative feedback system provides the self-gain module with the ability of self-adjusting. Experimental results have shown that the fluctuation of the output voltage is less than $0.1 \mathrm{~dB}$ at the range of $1 \mathrm{kHz}-10 \mathrm{MHz}$.

\section{Theory analysis}

It is easy to understand that a sinusoidal signal minus another sinusoidal signal in the same frequency produces a new sinusoidal signal. The amplitude of the new sinusoidal signal is decided by the phase shift and amplitudes of the former sinusoidal signals as shown in Fig. 2.

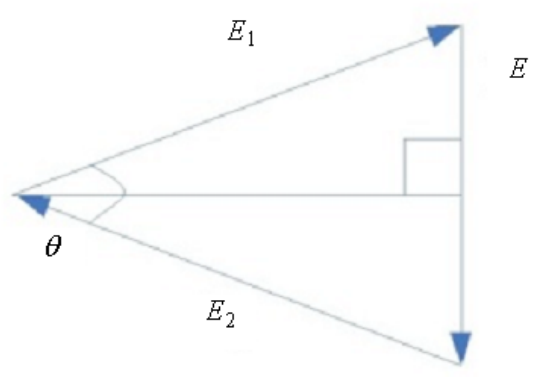

Fig. 2 Subtraction of two sinusoidal signals.

In this method, we assume that the amplitudes of the input sinusoidal signals are the same. It can be realized by a self-gain module as expressed in (2):

$$
E_{1}=E_{2}
$$

where $E_{1}$ and $E_{2}$ are the amplitudes of the input sinusoidal signals. In the isosceles triangle as shown in Fig. 2, the output sinusoidal signal after subtraction is expressed by vector $\mathbf{E}$. The phase shift between input sinusoidal signals $\theta$ can be expressed as (3):

$$
\sin \frac{\theta}{2}=\frac{E}{2 E_{1}}=\frac{E}{2 E_{2}}
$$

where $E$ is the amplitude of the output sinusoidal signal. Thus, the phase shift can be demodulated by

$$
\theta=2 \arcsin \left(\frac{E}{2 E_{1}}\right) .
$$

As shown in (4), the phase shift of two sinusoidal signals can be obtained by measuring the amplitude of the output sinusoidal signal after subtraction. The phase shift $\theta$ is proportional to the amplitude of the output sinusoidal signal. Referencing the theoretical analysis, we can learn that when the phase shift is less than 0.1 degree, the 
amplitude of the output signal will be so small to be submerged in the noise. As a result, we should amplify the output signal and eliminate the noises to improve the measurement accuracy.

\section{Phase shift demodulation system}

The phase shift demodulation system is conducted as shown in Fig. 3. Signals 1 and 2 are the input sinusoidal signals under measurement for the phase shift. A self-gain module is used to tune the amplitudes of the input sinusoidal signals into the same after which $E_{1}$ and $E_{2}$ discussed in Section 2 are achieved. A commercial chip AD8221 (Analog Devices, USA) is involved to build the subtraction circuit. In addition, a band-pass filter circuit constructed by UAF42 (Texas Instruments, USA) is used to improve the signal to noise ratio (SNR) of the output sinusoidal signal. Then, a good sinusoidal signal with high SNR from the subtracter is sent to a lock-in amplifier for the accurate measurement of amplitude $E$. The locked amplitude $E$ is collected by PC for some further process like (4), and then the phase shift $\theta$ between the input sinusoidal signals is demodulated.

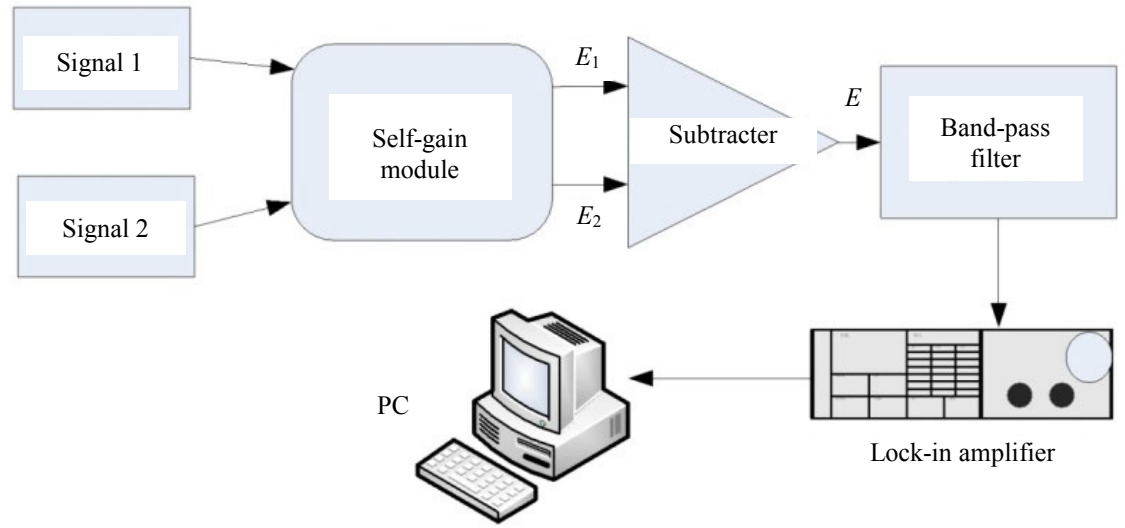

Fig. 3 Phase detection system.

\section{Functional verification}

\subsection{The designed LFDS}

In order to verify the performance of the phase shift demodulation system, it is easy for us to choose a signal generator to give the electric sinusoidal signals. But most of the signal generators are always incapable of providing signals in mini phase differential. As shown in Fig. 4, an optical source called laser fiber differential source (LFDS) is designed to give the infinitesimal phase angle in theory, whose basic principle is the light propagation in fiber in different lengths.

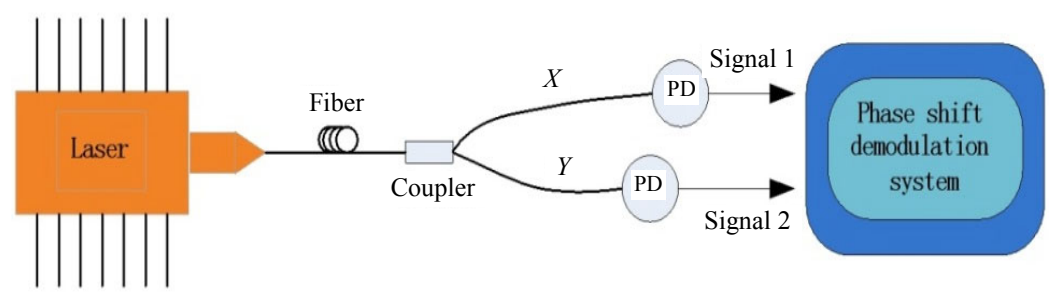

Fig. 4 Schematic of the LFDS.

In the LFDS, a distributed feedback laser diode driven by a $16 \mathrm{kHz}$ sinusoidal signal is used as the light source in the experiment. A 1:1 coupler is used to split the laser beam into two fibers in length of $X$ meter and $Y$ meter. The respective light is detected by the photo-detector, and the output signals can be 
expressed by Signals 1 and 2. Thus, a phase shift is generated between the output signals because of the optical path differences. The phase shift $\theta$ can be expressed as follows:

$$
\left\{\begin{array}{c}
\Delta l=X-Y \\
\varphi=\frac{\Delta l}{v} \\
\theta=2 \pi f \varphi=2 \pi f \frac{X-Y}{v}
\end{array}\right.
$$

where $X$ and $Y$ are the lengths of different fibers, $v$ is the light speed propagating in the fiber, and $f$ is the frequency of the laser driving signal which is set as $16 \mathrm{kHz}$ in the experiment. Obviously, the phase shift $\theta$ is related to the optical path differences $X-Y$ and laser modulation frequency $f$. In the experiment, it is simple but effective for us to get different phase shifts by changing the fiber length $X$ or $Y$. In theory, one meter of the optical path difference corresponds to 0.03 phase degree approximately. To verify the reliability of the LFDS in the generation of the phase shift, a lock-in amplifier Model 7230 (Ametek advanced measurement technology, USA) is used to calibrate the results of the LFDS as shown in Table 1 .

Table 1 Calibration of the LFDS by Model 7230.

\begin{tabular}{c|c|c|c|c}
\hline$\Delta l(\mathrm{~m})$ & $\theta$ (theory) & $\theta$ (Time 1) & $\theta$ (Time 2) & $\theta$ (Time 3) \\
\hline 1.04 & 0.03 & 0.03 & 0.03 & 0.03 \\
\hline 2.08 & 0.06 & 0.06 & 0.06 & 0.07 \\
\hline 3.12 & 0.09 & 0.09 & 0.10 & 0.09 \\
\hline 4.16 & 0.12 & 0.12 & 0.12 & 0.12 \\
\hline
\end{tabular}

The results in Table 1 reveal that the LFDS is reliable in mini phase shift production after multiple verifications.

\subsection{Functional verification of the phase shift demodulation system}

The mini phase shift can be generated by the LFDS as discussed in Section 4.1. Electric signals in a wide angle from 1 degree to 16 degree are produced by a signal generator. Our designed phase shift demodulation system is used to measure these signals in different phase angles from either the LFDS or the signal generator. The experimental results are plotted in Fig. 5.

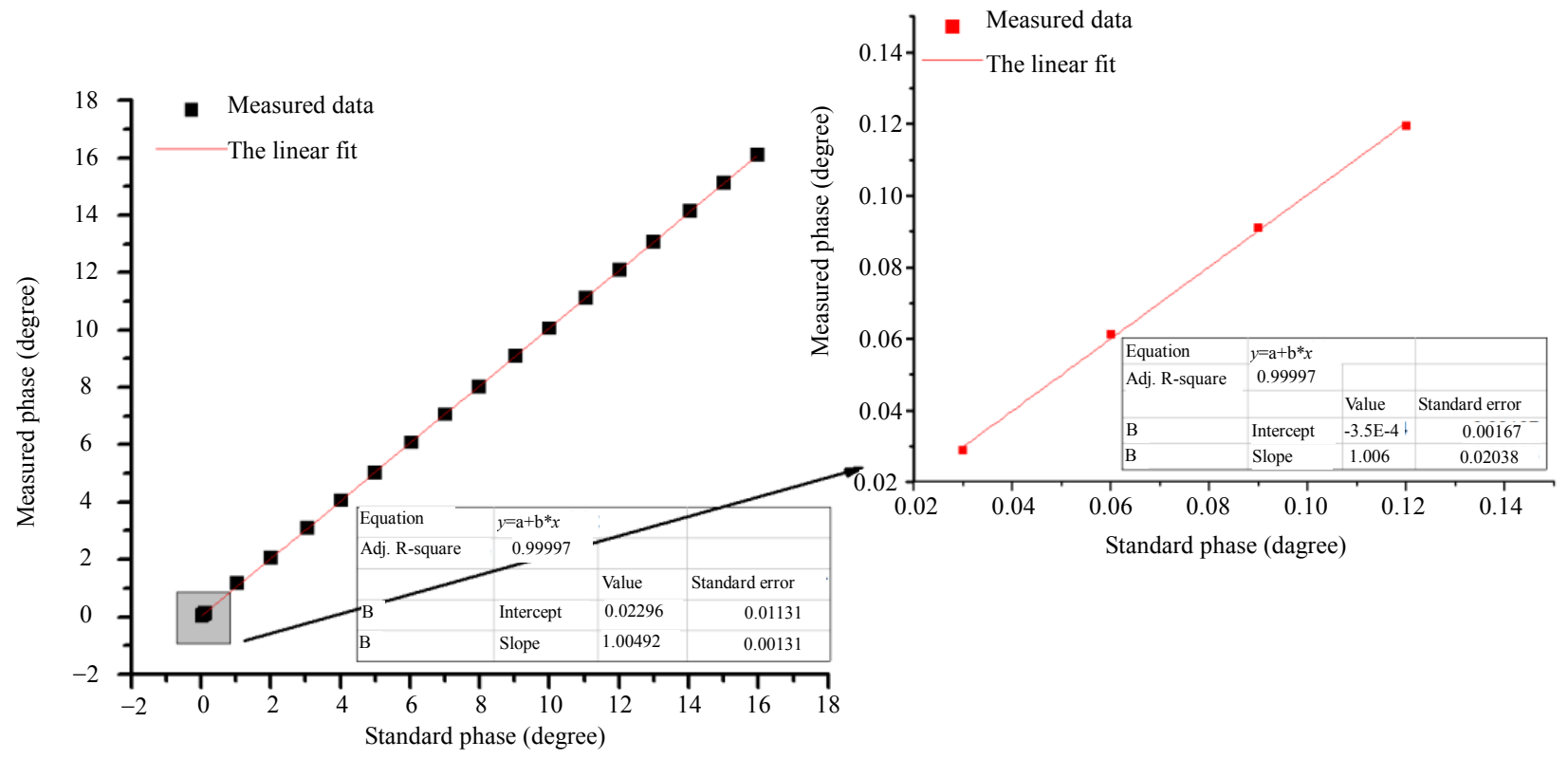

Fig. 5 Measured phases by the phase shift demodulation system.

A linear fit is applied to the measured data from

0.03 degree to 16 degree, and an R-square of 0.99997 
is achieved as shown in Fig. 5. In addition, we plot the phase data 0.03 degree, 0.06 degree, 0.09 degree, and 0.12 degree from the LFDS separately in the right inner figure which applies a linear fit too. The $\mathrm{R}$-square is 0.99877 . So, we can draw a conclusion that our self-designed phase shift demodulation system is reliable and the new phase shift demodulation technique is feasible.

\section{Application in fluorescence phase based oxygen sensor}

The purpose of designing the phase shift demodulation system is for some practical applications. Simultaneously, it is also necessary to verify it in the specific application. Oxygen sensing is of major importance in many fields, and phase shift measurement is always the crucial step in the fluorescence phase based oxygen sensor. In the oxygen concentration detection, the fluorescence intensity $I$ and lifetime $\tau$ of the sensing dye are described by the Stern-Volmer equation [7-9]:

$$
\frac{I_{0}}{I}=\frac{\tau_{0}}{\tau}=1+K_{\mathrm{SV}}\left[O_{2}\right]
$$

where $I_{0}$ and $\tau_{0}$ are the unquenched luminescence intensity and lifetime, respectively, $K_{\mathrm{SV}}$ is the Stern-Volmer constant, and $\mathrm{O}_{2}$ is the concentration of oxygen. Meanwhile, the phase difference between the exciting light signal and the fluorescence signal can be described by (7) if we apply a sinusoidal modulation signal to excitation source [10]:

$$
\tan \theta=2 \pi f \tau
$$

where $\theta$ is the phase shift between the exciting light and the fluorescence, $f$ is the frequency of the sinusoidal modulation, and $\tau$ is the lifetime of the fluorescence signal. Obviously, the phase shift demodulation system can be used to measure the fluorescence phase $\theta$ in the oxygen sensor as shown in Fig. 6.

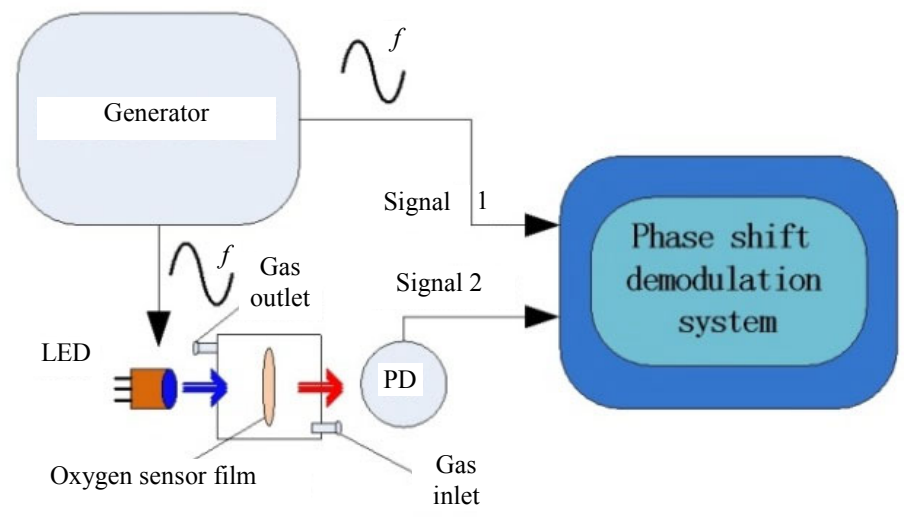

Fig. 6 Fluorescence-based optical oxygen sensor.

A sinusoidal signal with the frequency of $16 \mathrm{kHz}$ is used to drive the light-emitting diode (LED) to excite the oxygen sensor film, and another sinusoidal signal in the same frequency is generated as Signal 1. The fluorescence signal is transformed by the photo-detector into Signal 2 whose phase shift $\theta$ is proportional to the concentration of oxygen. Sample gas in different oxygen concentrations is accessed for measurement. The fluorescence phases in the concentration of $0.99 \%, 3 \%, 5 \%$, and $8 \%$ are measured by our phase shift demodulation system, and the results are plotted in Fig. 7. It is clear that the measured data display the good SNR and repeatability. A phase detection accuracy of 0.03 degree is achieved in $0.99 \%$ oxygen, and the oxygen concentration detection accuracy better than $0.02 \%$ is realized by virtue of our phase shift demodulation system. 


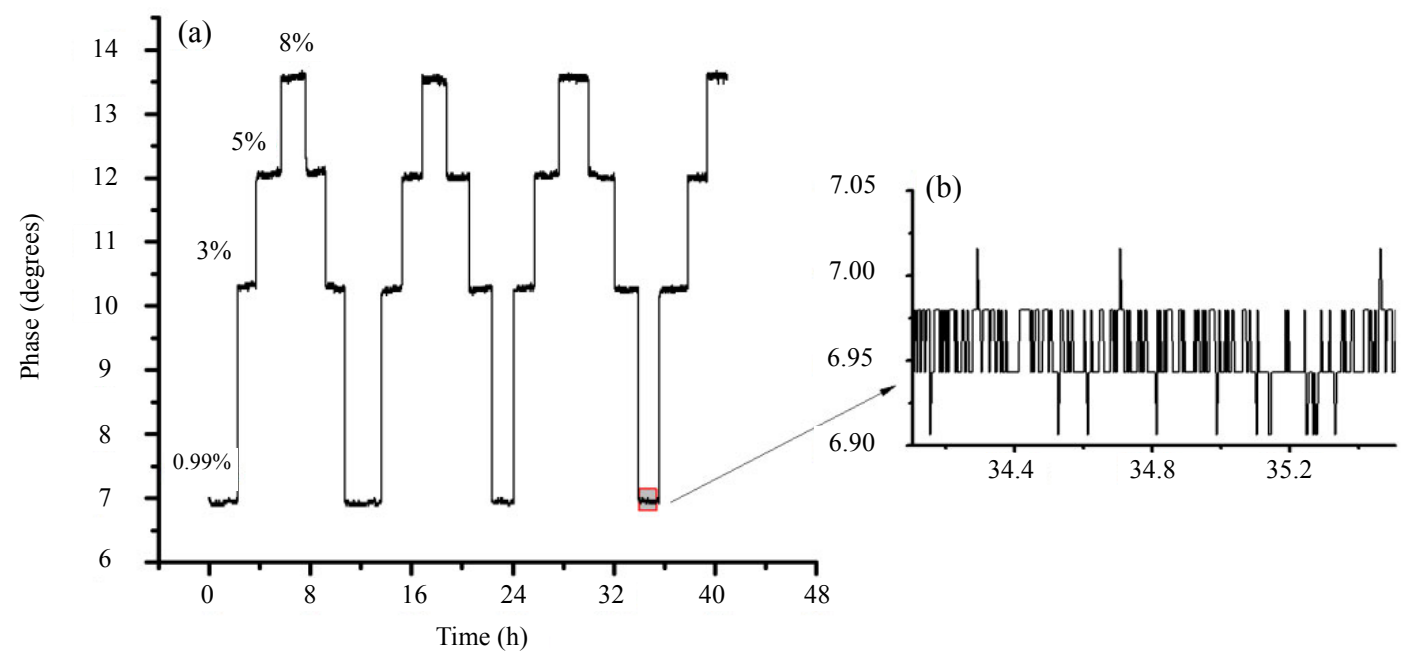

Fig. 7 Oxygen measurement in different sample gases with the concentration of $0.99 \%, 3 \%, 5 \%$, and $8 \%$ oxygen by our phase shift demodulation system.

\section{Conclusions}

A new phase shift demodulation system based on the self-gain module and subtraction is presented in this paper. Electric signals in the wide angle from 1 degree to 16 degree produced by the signal generator and mini phase shift generated by the LFDS are specially chosen to verify the function of the system. The experimental results show that R-square of 0.99997 in the electric signals and R-square of 0.99877 in the LFDS are achieved, and 0.03 degree measurement limit is realized in the experiments. Furthermore, this phase shift demodulation system can be successfully applied in the fluorescence phase based oxygen sensors by virtue of the high stability and precision. Experimental results have demonstrated that a good repetition and better than $0.02 \%$ oxygen concentration measurement accuracy are realized. In conclusion, this phase shift demodulation system is reliable, practical, and stable. In addition, this phase detection system could be utilized in high-accuracy, high-precision portable measurement systems for sensing applications.

\section{Acknowledgement}

This work was supported by the National
Natural Science Foundation of China (61475085), Science and Technology Development Project of Shandong Province (2014GGX101007), and the Fundamental Research Funds of Shandong University (2014YQ011).

Open Access This article is distributed under the terms of the Creative Commons Attribution 4.0 International License (http://creativecommons.org/ licenses/by/4.0/), which permits unrestricted use, distribution, and reproduction in any medium, provided you give appropriate credit to the original author(s) and the source, provide a link to the Creative Commons license, and indicate if changes were made.

\section{References}

[1] H. Gheidi and A. Banai, "Phase-noise measurement of microwave oscillators using phase - shifterless delay-line discriminator," IEEE Transactions on Microwave Theory and Techniques, 2010, 58(2): 468-477.

[2] W. Zhu, S. Zhu, and S. Ye, "Phase locked positioning method in heterodyne interferometer system," Opto-Electronic Engineering, 2014, 41(5): 35-39.

[3] G. Peng, M. Tang, H. Zou, C. Tian, and X. Chen, "Determination of TDC using digital correlation method for linear compressor," Journal of the Institute of Brewing, 2003, 694-697(5): 1608-1614.

[4] F. Chu, H. Cai, R. Q. Rong-Hui, Z. Fang, and J. Yang, "Study on optical fiber dissolved oxygen sensor based on fluorescence quenching," Journal of 
Optoelectronics Laser, 2009, 20(8): 1070-1072.

[5] J. Jia and M. Wang, "An optimisation method for the over-zero switching scheme," Flow Measurement and Instrumentation, 2012, 27(10): 47-52.

[6] X. Liu, X. Xu, and X. Tan, "The design of automatic gain control in self-excitation drive circuit of gyroscope," Chinese Journal of Sensors and Actuators, 2007, 26(11): 1662-1666.

[7] A. Campbell and D. G. Uttamchandani, "Optical dissolved oxygen lifetime sensor based on sol-gel immobilisation," IEE Proceedings Science Measurement and Technology Technology, 2004, 151(4): 291-297.

[8] X. Li, X. Lu, X. Luo, and G. Liu, "Development of the fluorescence quenching dissolved oxygen sensor," Automation \& Instrumentation, 2013, 28(4): 17-20.

[9] L. Pirrami, J. Wicht, F. Debrot, A. Rosspeintner, E. Vauthey, J. N. Aebischer, et al., "Oxygen sensor for indirect calorimetry bsaed on ruthenium fluorescence quenching," in IEEE 2011 International Semiconductor Conference, Sinaia, pp. 123-126, 2011.

[10] S. M. Borisov, G. Neuauter, C. Schroeder, I. Klimant, and O. S. Wolfbeis, "Modified dual lifetime referencing method for simultaneous optical determination and sensing of two analytes," Applied Spectroscopy, 2006, 60(10):1167-1173. 